\title{
A CONSTRUCTIVE ANALYSIS OF A PROOF THAT THE NUMERICAL RANGE IS CONVEX
}

\author{
DOUGLAS BRIDGES AND ROBIN HAVEA
}

\begin{abstract}
It is shown where the classical proof of the convexity of the numerical range of an operator on a Hilbert space breaks down by using principles that are not valid in intuitionistic logic. Those breakdowns are then repaired, as far as possible, to provide constructive versions of the convexity theorem. Finally, it is shown that our results are the best possible in a constructive setting.
\end{abstract}

\section{Introduction}

The classical Toeplitz-Hausdorff theorem says that if $T$ is a bounded linear operator on a Hilbert space $H$, then its numerical range

$$
W(T)=\{\langle T x, x\rangle: x \in H,\|x\|=1\}
$$

is convex. In his discussion of the proof of this theorem, Halmos [6, pp. 317-318] comments that

'Every known proof [of Theorem 1] is computational.'

Ironically, his proof is not really computational; for example, it cannot be translated directly into the language of recursive function theory. Our plan in this paper is to show

- where the proof given by Halmos (a proof he ascribes to C. de Boor) breaks down constructively by requiring some intuitionistically invalid logical principle, and

- to what extent those breakdowns can be repaired using intuitionistic logic.

The end-product of our analysis is the provision of fully constructive proofs of the following results. (Note, however, that the statement 'Every bounded operator on a Hilbert space has an adjoint' cannot be proved constructively [5].)

Theorem 1. Let $T$ be a selfadjoint operator on a Hilbert space $H$, let $x, y$ be unit vectors in $H$ such that $\langle T x, x\rangle \neq\langle T y, y\rangle$, and let

$$
h(t)=t x+(1-t) y \quad(0 \leqslant t \leqslant 1) .
$$

Then for each $c \in[0,1]$ there exists $t \in[0,1]$ such that

$$
\left\langle T\left(\|h(t)\|^{-1} h(t)\right),\|h(t)\|^{-1} h(t)\right\rangle=c\langle T x, x\rangle+(1-c)\langle T y, y\rangle .
$$

Theorem 2. If $T$ is a bounded operator on $H$ with an adjoint, then the closure of $W(T)$ is convex. 
We apply the word constructive exclusively to mathematics that uses intuitionistic logic and is based on principles-for example, those of intuitionistic Zermelo-Fraenkel set theory - that do not entail logical propositions, such as the law of excluded middle, that lie outside the scope of intuitionistic logic; see [9,2]. One feature of a constructive proof is that it can readily be translated into the language of recursive mathematics [8] or computable analysis [12]. (There are situations where, using an appropriate translation, a classical proof can routinely be converted into a constructive one. For example, this is the situation with so-called negative formulae in classical predicate logic; see [11, pp. 56-68].)

For background information about constructive analysis we refer the reader to $[\mathbf{1 , 4 , 1 1 ]}$. In practice, our paper requires almost no knowledge of constructive mathematics beyond an appreciation that we cannot, constructively, compare real numbers as freely as is possible (and trivial) using classical logic.

We begin by outlining Halmos's proof of the Toeplitz-Hausdorff theorem. Given unit vectors $x, y$ in $H$, write $\xi=\langle T x, x\rangle, \eta=\langle T y, y\rangle$. We must show that $t \xi+(1-t) \eta \in$ $W(T)$ for each $t \in[0,1]$. To this end, we split the proof into a number of steps as follows.

(i) Assuming that $\xi \neq \eta$, reduce to the case where $\xi=1$ and $\eta=0$.

(ii) Writing $B=\frac{1}{2 \mathrm{i}}\left(T-T^{*}\right)$, further reduce to the case where $\langle B x, x\rangle=0=\langle B y, y\rangle$ and $\operatorname{Re}\langle B x, y\rangle=0$.

(iii) Writing

$$
h(t)=t x+(1-t) y \quad(0 \leqslant t \leqslant 1),
$$

observe that as the vectors $x, y$ are linearly independent, $h(t)$ never vanishes.

(iv) Expanding $\langle B h(t), h(t)\rangle$, show that $\langle T h(t), h(t)\rangle \in \mathbf{R}$ for each $t \in[0,1]$. Hence the function

$$
t \mapsto \frac{\langle T h(t), h(t)\rangle}{\|h(t)\|^{2}}
$$

is real-valued and continuous on $[0,1]$. Since its values at 0 and 1 are, respectively, 0 and 1 , we conclude from the intermediate value theorem that the range of this function is $[0,1]$.

What, then, are the problems with the foregoing steps? In Step (i) we cannot expect to decide, for any complex numbers $\xi$ and $\eta$, that $\xi=\eta$ or $\xi \neq \eta$. (Two vectors $x, y$ are distinct, which we signify by $x \neq y$, if $\|x-y\|>0$.) The problem with Step (ii) occurs where Halmos multiplies a certain complex number $z$ by a complex number $\lambda$ of unit modulus to obtain $\operatorname{Re}(\lambda z)=0$; perhaps surprisingly, this cannot be done constructively for a general $z \in \mathbf{C}$. In Step (iii) we need to prove, for each $t \in[0,1]$, not just that $\neg(h(t)=0)$ but that $h(t) \neq 0$ in the stronger sense that $\|h(t)\|>0$; note that this sense is stronger unless we are prepared to accept the constructively dubious principle known as Markov's principle see [4, pp. 137-138].

One of the biggest problems with Halmos's proof occurs at the end, with the application of the intermediate value theorem: the best conclusion we have, constructively, using Halmos's argument as it stands, is that the range of the mapping $t \mapsto\|h(t)\|^{-2}\langle T h(t), h(t)\rangle$ is dense in $[0,1]$, which only enables us to assert that the closure of the segment joining $\langle T x, x\rangle$ and $\langle T y, y\rangle$ lies in $W(T)$. For the conclusion of the classical intermediate value theorem to hold constructively, the continuous function must satisfy one of a number of additional hypotheses, one of which is that the function be a polynomial, as follows. 
Proposition 3. If $f:[0,1] \rightarrow \mathbf{R}$ is a polynomial function such that $f(0)<0$ and $f(1)>0$, then there exists $x \in[0,1]$ such that $f(x)=0$ (see $[1, p .63$, Problem 17]).

Our proof of Theorem 1 depends on our ability to show that if $-1 \leqslant a \leqslant 1,0 \leqslant c \leqslant 1$, and $b \in \mathbf{R}$, then the quadratic equation

$$
(2 c(1-a)+2 b-1) t^{2}-2(c(1-a)+b) t+c=0
$$

has a root in $[0,1]$. (It is surely rare to find the humble quadratic equation playing such a major part in a modern research paper!) For convenience we set

$$
\begin{aligned}
\alpha & =c(1-a)+b, \\
p_{c}(t) & =(2 \alpha-1) t^{2}-2 \alpha t+c,
\end{aligned}
$$

so that equation (1) is just $p_{c}(t)=0$. We also write

$$
\begin{aligned}
& t_{-}=\frac{\alpha-\sqrt{\alpha^{2}-2 \alpha c+c}}{2 \alpha-1}, \\
& t_{+}=\frac{\alpha+\sqrt{\alpha^{2}-2 \alpha c+c}}{2 \alpha-1}
\end{aligned}
$$

for the standard solutions of equation (1) in the case $2 \alpha \neq 1$.

\section{Locating a root of $p_{c}(t)$ in $[0,1]$}

To set the scene for this section, we first observe that the law of trichotomy

$$
\forall x \in \mathbf{R}(x>0 \vee x=0 \vee x<0)
$$

cannot be proved constructively (see [4, pp. 3-4]). This is hardly surprising when we consider that constructive proofs have computational interpretations: we can always feed a real computer a number that, although nonzero, is too small for the computer to distinguish it from 0; this is the numerical analyst's problem of underflow.

How does this affect our subsequent constructive analysis? A typical situation that we have to deal with below is one in which, for a certain parametrised family $\left(p_{x}\right)_{x \geqslant 0}$ of polynomials, we know that the equation $p_{x}(t)=0$ has a solution if either $x>0$ or $x=0$. Since we cannot assert that for all $x \geqslant 0$ either $x>0$ or $x=0$, we have to adopt a subtle argument that, for a given $x \geqslant 0$, creates a Cauchy sequence of approximate solutions to the equation $p_{x}(t)=0$, the limit of which is an exact solution. The construction of the Cauchy sequence depends on the constructively valid proposition that if $a, b$ are real numbers with $a<b$, then for each real number $x$, either $x>a$ or $x<b$. Here is our first application of this technique.

Lemma 4. Let $I=[0,1]$, and let $\left(f_{c}\right)_{c \in I}$ be a family of mappings of I into $\mathbf{R}$ such that $f_{0}(0)=0$. Suppose that there exist strictly decreasing sequences $\left(\delta_{n}\right)_{n=1}^{\infty},\left(\varepsilon_{n}\right)_{n=1}^{\infty}$ in $(0,1)$ converging to 0 , such that if $0<x \leqslant \delta_{n}$, then $f_{x}(t)=0$ for some $t \in\left[0, \varepsilon_{n}\right]$. Then for each $c \in\left[0, \delta_{1}\right)$ there exists $t \in I$ such that $f_{c}(t)=0$.

Proof. Given $c \in\left[0, \delta_{1}\right)$, construct an increasing binary sequence $\left(\lambda_{n}\right)$ such that

$$
\begin{aligned}
& \lambda_{n}=0 \Rightarrow c<\delta_{n+1}, \\
& \lambda_{n}=1 \Rightarrow c>\delta_{n+2} .
\end{aligned}
$$


We may assume that $\lambda_{1}=0$. If $\lambda_{n}=0$, choose $t_{n} \in\left[0, \varepsilon_{n+1}\right]$ such that $f_{\delta_{n+1}}\left(t_{n}\right)=0$. If $\lambda_{n}=1-\lambda_{n-1}$, then $\delta_{n+2}<c<\delta_{n}$, and so there exists $t_{n} \in\left[0, \varepsilon_{n}\right]$ such that $f_{c}\left(t_{n}\right)=0$; in this case we set $t_{k}=t_{n}$ for all $k \geqslant n$. Then $\left(t_{n}\right)$ is a Cauchy sequence in [0,1]; in fact, $\left|t_{m}-t_{n}\right|<2 \varepsilon_{n}$ for all $n \geqslant 2$. Hence $\left(t_{n}\right)$ converges to a limit $t_{\infty} \in\left[0, \varepsilon_{1}\right] \subset I$. If $f_{c}\left(t_{\infty}\right) \neq 0$, then $\lambda_{n}=0$ for all $n$, so $c=0=t_{\infty}$ and therefore $f_{c}\left(t_{\infty}\right)=f_{0}(0)=0$, a contradiction. Hence, in fact, $f_{c}\left(t_{\infty}\right)=0$.

The following lemma is not quite as trivial as may at first appear, for if $p(x)$ is a monic quadratic polynomial with roots $t_{1}, t_{2}$ given by the standard formula, then $\max \left\{t_{1}, t_{2}\right\}$ and $\min \left\{t_{1}, t_{2}\right\}$ also satisfy $p(x)=0$, and we may be unable to decide whether $\max \left\{t_{1}, t_{2}\right\}=t_{1}$ or $\max \left\{t_{1}, t_{2}\right\}=t_{2}$. It is, of course, impossible for a quadratic equation to have three distinct roots.

Lemma 5. Let $p(x)$ be a monic quadratic polynomial with real roots $t_{1}$, $t_{2}$ given by the standard formula. Then $|t| \leqslant \max \left\{\left|t_{1}\right|,\left|t_{2}\right|\right\}$ for any real root $t$ of $p$.

Proof. Write $p(x)=x^{2}+\beta x+\gamma$. Let $t$ be a root of $p$, so that

$$
p(x)=(x-t)\left(x-t^{\prime}\right)
$$

where $t+t^{\prime}=-\beta$ and $t t^{\prime}=\gamma$. Suppose that $|t|>\max \left\{\left|t_{1}\right|,\left|t_{2}\right|\right\}$. Then since

$$
\left(|t|+\left|t^{\prime}\right|\right)^{2}=\beta^{2}+2(|\gamma|-\gamma)=\left(\left|t_{1}\right|+\left|t_{2}\right|\right)^{2},
$$

we have

$$
|t|+\left|t^{\prime}\right|=\left|t_{1}\right|+\left|t_{2}\right| \leqslant 2 \max \left\{\left|t_{1}\right|,\left|t_{2}\right|\right\}
$$

whence $\left|t^{\prime}\right|<\max \left\{\left|t_{1}\right|,\left|t_{2}\right|\right\}$. This leads to the absurd conclusion that $p(x)$ has three distinct roots: namely, $t, t^{\prime}$, and at least one of $t_{1}$ and $t_{2}$. Hence $|t| \leqslant \max \left\{\left|t_{1}\right|,\left|t_{2}\right|\right\}$.

Lemma 6. For each $\varepsilon>0$ there exists $\delta>0$ such that if $-1 \leqslant a \leqslant 1,|b|<\delta, 0 \leqslant c<\delta$, and $t$ is a real root of equation (1), then $|t|<\varepsilon$.

Proof. Recalling our introduction of $\alpha$ at the end of Section 1, we see that if $0<\delta<1$, $|b|<\delta$, and $0 \leqslant c<\delta$, then

$$
|\alpha|<\delta(1-a)+\delta \leqslant 3 \delta
$$

and therefore

$$
\begin{aligned}
\left|\alpha^{2}-2 \alpha c+c\right| & \leqslant|\alpha|^{2}+2|\alpha| c+c \\
& <9 \delta^{2}+6 \delta^{2}+\delta \\
& <16 \delta .
\end{aligned}
$$

If also $0<\delta \leqslant 1 / 12$, then $|2 \alpha-1| \geqslant 1-2|\alpha|>1 / 2$, and so the solutions $t_{-}, t_{+}$of the quadratic equation (1) satisfy

$$
\max \left\{\left|t_{-}\right|,\left|t_{+}\right|\right\} \leqslant 2\left(|\alpha|+\left|\alpha^{2}-2 \alpha c+c\right|\right)<38 \delta \text {. }
$$

Using Lemma 5, we deduce that $|t|<38 \delta$ for all real roots $t$ of equation (1). Given $\varepsilon>0$, it remains to take $\delta=\min \left\{\frac{1}{12}, \frac{\varepsilon}{38}\right\}$. 
Lemma 7. Let $-1 \leqslant a \leqslant 1$ and $b<0 \leqslant c<\frac{1}{4}$. If $|b|>3 c / 2$, then $0<t_{-}<1$.

Proof. First note that

$$
2 \alpha-1=2 c(1-a)+2 b-1<4 c-3 c-1<-\frac{3}{4},
$$

that

$$
\alpha-c=-c a+b<c+b<0,
$$

and that

$$
2 \alpha-c=2 b+2 c(1-a)-c<-3 c+4 c-c=0 .
$$

Since

$$
\alpha^{2}-2 \alpha c+c \geqslant \alpha^{2}-2 \alpha c+c^{2}=(\alpha-c)^{2},
$$

it follows that $\sqrt{\alpha^{2}-2 \alpha c+c}$ is real,

that

$$
\alpha-\sqrt{\alpha^{2}-2 \alpha c+c} \leqslant \alpha-(c-\alpha)=2 \alpha-c,
$$

and therefore that

$$
t_{-}=\frac{\alpha-\sqrt{\alpha^{2}-2 \alpha c+c}}{2 \alpha-1} \geqslant \frac{2 \alpha-c}{2 \alpha-1}>0 .
$$

On the other hand, since $0 \leqslant c \leqslant 1 / 4$ and $2 \alpha-1<0$,

$$
\begin{aligned}
0 & \leqslant \alpha^{2}-2 \alpha c+c \\
& =\alpha^{2}-2 c\left(\alpha-\frac{1}{2}\right) \\
& <\alpha^{2}-\frac{1}{2}\left(\alpha-\frac{1}{2}\right)=\left(\alpha-\frac{1}{2}\right)^{2}
\end{aligned}
$$

and therefore

$$
\sqrt{\alpha^{2}-2 \alpha c+c}<\frac{1}{2}-\alpha
$$

Hence

$$
t_{-}<\frac{2 \alpha-\frac{1}{2}}{2 \alpha-1}
$$

and therefore $t_{-}<1$.

Lemma 8. If $-1 \leqslant a \leqslant 1, b>0$, and $0 \leqslant c<1$, then $p_{c}(t)=0$ for some $t \in[0,1)$.

Proof. Since $b$ is positive, so is $\alpha$. Hence for every $c \in(0,1)$,

$$
p_{c}\left(c^{1 / 2}\right)=(2 \alpha-1) c-2 \alpha c^{1 / 2}+c=2 \alpha\left(c-c^{1 / 2}\right)<0 .
$$

But $p_{c}(0)=c>0$; so, by Proposition 3, there exists $t \in\left(0, c^{1 / 2}\right)$ with $p_{c}(t)=0$. We now invoke Lemma 4 to show that for each $c \in[0,1)$ there exists $t \in[0,1)$ such that $p_{c}(t)=0$.

Proposition 9. Let $-1 \leqslant a \leqslant 1, b \in \mathbf{R}$, and $0 \leqslant c \leqslant 1$. Then $p_{c}(t)=0$ for some $t$ in $[0,1]$. 
Proof. Given $c \in[0,1]$, since $p_{c}(0)=c$ and $p_{c}(1)=c-1$, we see from Proposition 3 that if $0<c<1$, then there exists $t \in(0,1)$ such that $p_{c}(t)=0$. Clearly, $p_{c}(t)=0$ has a solution $t \in[0,1]$ if $c=0$ or $c=1$. But what if, as can happen in the constructive context, $c$ is close to, but not necessarily distinguishable from, one of the numbers 0,1 ? We consider only the case where $0 \leqslant c<1$, as the other case, $0<c \leqslant 1$, is handled similarly.

In view of Lemmas 7 and 8 , we need only deal with what happens when $b$ is also near 0 . To this end we use Lemma 6 to construct a strictly decreasing sequence $\left(\delta_{n}\right)_{n=1}^{\infty}$ of positive numbers converging to 0 , with $\delta_{1}<1 / 4$, such that if $|b|<\delta_{n}$ and $0 \leqslant c<\delta_{n}$, then $|t|<\frac{1}{n}$ for every solution $t$ of equation (1). Given $c \in[0,1)$, define an increasing binary sequence $\left(\lambda_{n}\right)$ such that

$$
\begin{aligned}
& \lambda_{n}=0 \Rightarrow|b|<\delta_{n} \text { and } c<\delta_{n+1}, \\
& \lambda_{n}=1 \Rightarrow|b|>\delta_{n+1} \text { or } c>\delta_{n+2} .
\end{aligned}
$$

We may assume that $\lambda_{1}=0$. If $\lambda_{n}=0$, set $t_{n}=0$. If $\lambda_{n}=1-\lambda_{n-1}$, then we have three cases to deal with. In the first case, $b<-\delta_{n+1}$ and so, by Lemma 7, there exists $t_{n} \in(0,1)$ such that $p_{c}\left(t_{n}\right)=0$; in the second case, $b>\delta_{n+1}$ and so, by Lemma 8 , there exists $t_{n} \in[0,1)$ such that $p_{c}\left(t_{n}\right)=0$; in the third case, $c>\delta_{n+2}$ and so, by the observation at the start of this proof, there exists a solution $t_{n}$ of equation $(1)$ in $(0,1)$. In each of these three cases, we set $t_{k}=t_{n}$ for all $k \geqslant n$, and we note that $\left|t_{n}\right|<1 /(n-1)$, since $\lambda_{n-1}=0$ and therefore $\max \{|b|, c\}<\delta_{n-1}$. This completes the inductive construction of $t_{n}$. Since $\left|t_{n}\right| \leqslant 1 /(n-1)$ for each $n \geqslant 2,\left(t_{n}\right)$ is a Cauchy sequence, and so converges to a limit $t_{\infty} \in[0,1]$. If $p_{c}\left(t_{\infty}\right) \neq 0$, then $\lambda_{n}=1$ for all $n$; whence $c=0=t_{\infty}$ and $p_{c}\left(t_{\infty}\right)=p_{0}(0)=0$, which is absurd. We conclude that $p_{c}\left(t_{\infty}\right)=0$.

\section{Proofs of the main results}

We are almost ready to prove Theorems 1 and 2, but we still require a couple of lemmas.

Lemma 10. Let $x, y$ be unit vectors in $H$, and for each $t \in[0,1]$ let $h(t)=t x+(1-t) y$. If $0 \leqslant t \leqslant \varepsilon<\frac{1}{2}$, then $\|h(t)\| \geqslant 1-2 \varepsilon$ and

$$
\|\| h(t)\left\|^{-1} h(t)-y\right\| \leqslant \frac{4 \varepsilon}{1-2 \varepsilon} .
$$

Proof. We have

$$
\begin{aligned}
\|h(t)\| & \geqslant(1-t)\|y\|-t\|x\| \\
& \geqslant 1-\varepsilon-\varepsilon \\
& =1-2 \varepsilon .
\end{aligned}
$$

Since $\|h(t)\| \leqslant 1$, it follows that $0 \leqslant 1-\|h(t)\|<2 \varepsilon$; whence

$$
\begin{aligned}
\|\| h(t)\left\|^{-1} h(t)-y\right\| & =\|h(t)\|^{-1}\|t x+(1-t) y-\| h(t)\|y\| \\
& \leqslant \frac{1}{1-2 \varepsilon}(t\|x-y\|+|1-\|h(t)\||\|y\|) \\
& \leqslant \frac{1}{1-2 \varepsilon}(2 \varepsilon+2 \varepsilon) \\
& =\frac{4 \varepsilon}{1-2 \varepsilon},
\end{aligned}
$$

as required. 
For our next lemma we recall that a mapping $f: X \rightarrow Y$ between metric spaces is sequentially continuous if $f\left(x_{n}\right) \rightarrow f(x)$ whenever $\left(x_{n}\right)$ is a sequence converging to $x$ in $X$; and that if $f$ is sequentially continuous and $X$ is complete, then $f$ is strongly extensional, in the sense that $f(x) \neq f(y)$ implies that $x \neq y$ (see [7, Theorem 1]).

Lemma 11. Let $T$ be a sequentially continuous operator on a Hilbert space $H$, let $x, y$ be unit vectors in $H$ such that $\langle T x, x\rangle \neq\langle T y, y\rangle$, and write

$$
h(t)=t x+(1-t) y \quad(0 \leqslant t \leqslant 1) .
$$

Then $h(t) \neq 0$ for every $t \in[0,1]$. Moreover, if $T$ is bounded, then $\inf _{t \in[0,1]}\|h(t)\|>0$.

Proof. Replacing $T$ by $T-\langle T y, y\rangle I$, where $I$ is the identity operator on $H$, we may assume that $\langle T y, y\rangle=0$. If $0<t<1$, then

$$
\left\langle T\left(\frac{t}{1-t} x\right), \frac{t}{1-t} x\right\rangle=\frac{t^{2}}{(1-t)^{2}}>0=\langle T(-y),-y\rangle ;
$$

so, as the quadratic form induced by $T$ is sequentially continuous, $(t /(1-t)) x \neq-y$ and therefore $t x+(1-t) y \neq 0$. If either $0 \leqslant t<\frac{1}{3}$ or $\frac{2}{3}<t \leqslant 1$, then Lemma 10 yields $\|h(t)\|>\frac{1}{3}$. Putting these three possibilities for $t$ together, we conclude that $h(t) \neq 0$ for all $t \in[0,1]$.

Now consider the case where $T$ is bounded and not just sequentially continuous. Choose $\delta>0$ such that if $\|z\| \leqslant 1,\left\|z^{\prime}\right\| \leqslant 1$, and $\left\|z-z^{\prime}\right\|<\delta$, then $\left|\langle T z, z\rangle-\left\langle T z^{\prime}, z^{\prime}\right\rangle\right|<1 / 9$. If $t \in\left[\frac{1}{4}, \frac{3}{4}\right]$, then

$$
\left\langle T\left(\frac{t}{1-t} x\right), \frac{t}{1-t} x\right\rangle=\frac{t^{2}}{(1-t)^{2}} \geqslant \frac{1}{9},
$$

so as $\langle T(-y),-y\rangle=0$, we have $\left\|\frac{t}{1-t} x+y\right\| \geqslant \delta$ and therefore $\|h(t)\| \geqslant(1-t) \delta \geqslant \frac{1}{4} \delta$. Taking this with the cases $0 \leqslant t<\frac{1}{3}$ and $\frac{2}{3}<t \leqslant 1$, we see that $\|h(t)\| \geqslant \min \left\{\frac{1}{3}, \frac{1}{4} \delta\right\}$.

Proof of Theorem 1. Let $x, y$ be unit vectors in $H$, and let $0 \leqslant c \leqslant 1$. We seek $t \in[0,1]$ such that

$$
\left\langle T\left(\|h(t)\|^{-1} h(t)\right),\|h(t)\|^{-1} h(t)\right\rangle=c\langle T x, x\rangle+(1-c)\langle T y, y\rangle .
$$

First, observe that since $\langle T x, x\rangle \neq\langle T y, y\rangle$, we can find scalars $\lambda, \mu$ such that $\langle(\lambda T+\mu I) x, x\rangle=1$ and $\langle(\lambda T+\mu I) y, y\rangle=0$; in that case

$$
c=\lambda(c\langle T x, x\rangle+(1-c)\langle T y, y\rangle)+\mu,
$$

so if there exists $t \in[0,1]$ such that

$$
\left\langle(\lambda T+\mu I)\left(\|h(t)\|^{-1} h(t)\right),\left(\|h(t)\|^{-1} h(t)\right)\right\rangle=c,
$$

then equation (2) holds. Thus we need only consider the case where

$$
\xi=\langle T x, x\rangle=1, \eta=\langle T y, y\rangle=0 .
$$

Writing

$$
h(t)=t x+(1-t) y \quad(0 \leqslant t \leqslant 1),
$$


$a=\operatorname{Re}\langle x, y\rangle$ and $b=\operatorname{Re}\langle T x, y\rangle$, and using routine computations with inner products, we see that

$$
c=\left\langle T\left(\|h(t)\|^{-1} h(t)\right),\|h(t)\|^{-1} h(t)\right\rangle=\|h(t)\|^{-2}\langle T h(t), h(t)\rangle
$$

if and only if $p_{c}(t)=0$. It now follows from Proposition 9 that there exists $t \in[0,1]$ such that $c\|h(t)\|^{2}=\langle T h(t), h(t)\rangle$. Since $T$, being selfadjoint, is sequentially continuous [3, Theorem 4], it follows from this and Lemma 11 that equation (2) holds with $z=$ $\|h(t)\|^{-1} h(t)$.

Corollary 12. Let $T$ be a bounded operator on a Hilbert space $H$, and let $x, y$ be unit vectors such that $\langle T x, x\rangle \neq\langle T y, y\rangle$ and $\langle T x, y\rangle \neq\left\langle T^{*} x, y\right\rangle$. Then for each $c \in[0,1]$ there exists a unit vector $z \in H$ such that $\langle T z, z\rangle=c\langle T x, x\rangle+(1-c)\langle T y, y\rangle$.

Proof. Write $T=A+\mathrm{i} B$ with $A=\frac{1}{2}\left(T+T^{*}\right)$ and $B=\frac{1}{2 \mathrm{i}}\left(T-T^{*}\right)$. Then $\langle B x, y\rangle \neq$ 0 , so (as in Step (ii) of Halmos's proof) there exists $\gamma \in \mathbf{C}$ such that $|\gamma|=1$ and $\operatorname{Re}\langle B(\gamma x), y\rangle=0$. Set

$$
h_{\gamma}(t)=t(\gamma x)+(1-t) y .
$$

Then (as on page 317 of [6]) $\left\langle B h_{\gamma}(t), h_{\gamma}(t)\right\rangle=0$ for all $t$; so (as in Step (iv) of Halmos's proof) $\left\langle T h_{\gamma}(t), h_{\gamma}(t)\right\rangle=\left\langle A h_{\gamma}(t), h_{\gamma}(t)\right\rangle$. Also, $\langle T x, x\rangle=\langle A(\gamma x), \gamma x\rangle$ and $\langle T y, y\rangle=$ $\langle A y, y\rangle$. Applying Theorem 1 with $A$ replacing $T$ and $\gamma x$ replacing $x$, for each $c \in[0,1]$ we obtain $t \in[0,1]$ such that

$$
\begin{aligned}
\left\langle T\left(\left\|h_{\gamma}(t)\right\|^{-1} h_{\gamma}(t)\right),\left\|h_{\gamma}(t)\right\|^{-1} h_{\gamma}(t)\right\rangle & =\left\langle A\left(\left\|h_{\gamma}(t)\right\|^{-1} h_{\gamma}(t)\right),\left\|h_{\gamma}(t)\right\|^{-1} h_{\gamma}(t)\right\rangle \\
& =c\langle A(\gamma x), \gamma x\rangle+(1-c)\langle A y, y\rangle \\
& =c\langle T x, x\rangle+(1-c)\langle T y, y\rangle,
\end{aligned}
$$

as required.

Proof of Theorem 2. Given that $\varepsilon>0$ and $c \in[0,1]$, we seek a unit vector $z$ such that

$$
|\langle T z, z\rangle-c\langle T x, x\rangle-(1-c)\langle T y, y\rangle|<\varepsilon .
$$

If $|\langle T x, x\rangle-\langle T y, y\rangle|<\varepsilon$, we may take $z=x$. Thus we may assume that $\langle T x, x\rangle \neq$ $\langle T y, y\rangle$.

First consider the case where $T$ is selfadjoint. Define a function $f:[0,1] \rightarrow \mathbf{R}$ by

$$
f(t)=\left|\left\langle T\left(\|h(t)\|^{-1} h(t)\right),\|h(t)\|^{-1} h(t)\right\rangle-c\langle T x, x\rangle-(1-c)\langle T y, y\rangle\right| .
$$

Since $T$ is bounded, it is straightforward to show, using Lemma 11, that $f$ is uniformly continuous on $[0,1]$; so

$$
m=\inf _{0 \leqslant t \leqslant 1} f(t)
$$

exists. It follows from Theorem 1 that if $m>0$, then $\neg(\langle T x, x\rangle \neq\langle T y, y\rangle)$ and therefore $\langle T x, x\rangle=\langle T y, y\rangle$; whence, trivially, $m=0$, a contradiction. We conclude that $m=0$; whence equation (3) holds with $z=\|h(t)\|^{-1} h(t)$ for some $t \in[0,1]$.

Now consider the general case. Write $T=A+\mathrm{i} B$, where $A=\frac{1}{2}\left(T+T^{*}\right)$ and $B=$ $\frac{1}{2 \mathrm{i}}\left(T-T^{*}\right)$ are bounded selfadjoint operators, and let $\varepsilon>0$. Noting that

$$
\langle A x, x\rangle=\langle T x, x\rangle \neq\langle T y, y\rangle=\langle A y, y\rangle
$$


and that $A$ is bounded, we see from Lemma 11 that

$$
0<r=\inf \{\|h(t)\|: 0 \leqslant t \leqslant 1\} .
$$

Either $\operatorname{Re}\langle B x, y\rangle \neq 0$ or $|\operatorname{Re}\langle B x, y\rangle|<r^{2} \varepsilon$. In the first case we apply Corollary 12 to obtain a unit vector $z$ such that equation (2), and therefore equation (3), holds. In the second case we apply the first part of the proof with $T$ replaced by $A$ to obtain $t \in[0,1]$ such that

$$
\left|\left\langle A\left(\|h(t)\|^{-1} h(t)\right),\|h(t)\|^{-1} h(t)\right\rangle-c\langle A x, x\rangle+(1-c)\langle A y, y\rangle\right|<\frac{1}{2} \varepsilon .
$$

Since

$$
\begin{gathered}
|\langle B h(t), h(t)\rangle|=2 t(1-t)|\operatorname{Re}\langle B x, y\rangle| \leqslant \frac{1}{2} r^{2} \varepsilon \\
\langle T x, x\rangle=\langle A x, x\rangle \quad \text { and } \quad\langle T y, y\rangle=\langle A y, y\rangle
\end{gathered}
$$

it follows that

$$
\begin{aligned}
& \left|\left\langle T\left(\|h(t)\|^{-1} h(t)\right),\|h(t)\|^{-1} h(t)\right\rangle-c\langle T x, x\rangle+(1-c)\langle T y, y\rangle\right| \\
\leqslant & \left|\left\langle A\left(\|h(t)\|^{-1} h(t)\right),\|h(t)\|^{-1} h(t)\right\rangle-c\langle A x, x\rangle+(1-c)\langle A y, y\rangle\right| \\
& \quad+\|h(t)\|^{-2}|\langle B h(t), h(t)\rangle| \\
< & \frac{1}{2} \varepsilon+r^{-2} \frac{1}{2} r^{2} \varepsilon \\
= & \varepsilon
\end{aligned}
$$

as we wanted.

\section{Weakening the hypotheses of Theorem 1}

It would be interesting, if not necessarily of great practical value, to remove from Theorem 1 the hypothesis that $\langle T x, x\rangle \neq\langle T y, y\rangle$. To show that we can do this under certain circumstances, we need a lemma that examines the behaviour of the roots of $p_{c}(t)$ in $[0,1]$ when $b$ is large and positive.

Lemma 13. For each positive integer $n$ there exists $K_{n}>0$, independent of the parameters $a$ and $c$ of $p_{c}$, such that if $b>K_{n}$, then $0 \leqslant t_{-} \leqslant \frac{1}{n}$.

Proof. Noting that $\alpha \geqslant b-2$, we see that if $b$ is large and positive, then so are $\alpha$ and $2 \alpha-1$, and also $\alpha^{2}-2 \alpha c+c<\alpha^{2}$; whence

$$
t_{-}=\frac{\alpha-\sqrt{\alpha^{2}-2 \alpha c+c}}{2 \alpha-1}>\frac{\alpha-\alpha}{2 \alpha-1}=0 .
$$

Also, since for such $b$,

$$
\alpha^{2}-2 \alpha c+c \geqslant \alpha^{2}-2 \alpha c^{1 / 2}+c=\left(\alpha-c^{1 / 2}\right)^{2}
$$

we have

$$
t_{-}<\frac{\alpha-\left(\alpha-c^{1 / 2}\right)}{2 \alpha-1}=\frac{c^{1 / 2}}{2 \alpha-1} \leqslant \frac{1}{2 b-5} .
$$

Thus it is enough to set $K_{n}=(n+5) / 2$. 
Returning to Theorem 1 , let us remove the hypothesis that $\langle T x, x\rangle \neq\langle T y, y\rangle$, and instead assume that $\langle T x, y\rangle \neq\langle T y, y\rangle\langle x, y\rangle$. Write

$$
\begin{gathered}
\delta=|\langle T x, y\rangle-\langle T y, y\rangle\langle x, y\rangle|>0, \\
\xi=\langle T x, x\rangle \text { and } \eta=\langle T y, y\rangle .
\end{gathered}
$$

Replacing $T$ by $T-\eta I$, if necessary, we may assume that $\eta=0$. Fix $c \in[0,1]$. With $K_{n}$ as in Lemma 13, construct an increasing binary sequence $\left(\lambda_{n}\right)_{n=1}^{\infty}$ such that

$$
\begin{aligned}
& \lambda_{n}=0 \quad \Rightarrow \quad|\xi|<K_{n}^{-1} \delta, \\
& \lambda_{n}=1 \quad \Rightarrow \quad|\xi|>K_{n+1}^{-1} \delta .
\end{aligned}
$$

If $\lambda_{2}=1$, then we are back in the case already covered by Theorem 1; so we may assume that $\lambda_{2}=0$. If $\lambda_{n}=0$, set $z_{n}=y$. If $\lambda_{n}=1-\lambda_{n-1}$, then $n \geqslant 3$ and we can choose $\gamma \in \mathbf{C}$ such that $|\gamma|=1$ and

$$
b=\operatorname{Re}\left(\xi^{-1}\langle T(\gamma x), y\rangle\right)=|\xi|^{-1} \delta>K_{n} .
$$

Writing $h_{\gamma}(t)=t(\gamma x)+(1-t) y$, and applying Lemma 13 with $\xi^{-1} T$ replacing $T$ and $\gamma x$ replacing $x$, we compute $t \in\left[0, \frac{1}{n}\right]$ such that

$$
z=\left\|h_{1}(t)\right\|^{-1} h_{1}(t)
$$

satisfies

$$
\langle T z, z\rangle=c\langle T(\gamma x), \gamma x\rangle=c \xi
$$

Setting $z_{k}=z$ for all $k \geqslant n$, we see from Lemma 10 that

$$
\|h(t)\|>1-\frac{2}{n} \geqslant \frac{1}{3}
$$

and $\left\|z_{k}-y\right\| \leqslant \frac{4}{n-2}$. This completes the construction of a sequence $\left(z_{n}\right)$ of unit vectors in $H$.

Since $\left\|z_{m}-z_{n}\right\| \leqslant \frac{8}{n-2}$ whenever $m \geqslant n \geqslant 3,\left(z_{n}\right)$ is a Cauchy sequence and therefore converges to a unit vector $z_{\infty} \in H$. Suppose that $\left\langle T z_{\infty}, z_{\infty}\right\rangle \neq c \xi$. Then $\lambda_{n}=0$ for all $n$; whence $\xi=0, z_{\infty}=y$, and

$$
\left\langle T z_{\infty}, z_{\infty}\right\rangle=\langle T y, y\rangle=0=c \xi,
$$

a contradiction. It follows that $c \xi=\left\langle T z_{\infty}, z_{\infty}\right\rangle \in W(T)$.

\section{Limiting examples}

We end the paper with two Brouwerian examples which show that our main results, and the weakening of the hypotheses of Theorem 1 that we have just discussed, are the best possible in the constructive setting. For these examples (which we state as propositions) we need to appreciate that for each positive integer $N$ the following omniscience principle is an essentially nonconstructive result. (For example, $\mathrm{LLPO}_{2}$ cannot be derived within Heyting arithmetic and is false, even classically, in the recursive model of constructive mathematics; see [4, Chapters 1, 3 and 7].) 
$\mathbf{L L P O}_{N}$ : If $\left(a_{n}\right)$ is a binary sequence with at most one term equal to 1 , then there exists $j$, where $0 \leqslant j \leqslant N-1$, such that $a_{k N+j}=0$ for all $k$.

$($ See $[10, \S 3]$.

The principle $\mathrm{LLPO}_{2}$ is known as the lesser limited principle of omniscience, and is usually denoted by LLPO. It implies the intermediate value theorem in the following form.

LLPO: If $f$ is a continuous mapping on $[0,1]$ such that $f(0) f(1) \leqslant 0$, then there exists $t \in[0,1]$ such that $f(t)=0$.

The proof is a standard interval-halving argument, based on the observation that LLPO implies (it is actually equivalent to) the proposition

$$
\forall x \in \mathbf{R}(x \geqslant 0 \vee x \leqslant 0) .
$$

Now let $T$ be a selfadjoint operator on $H$, and let $x, y$ be unit vectors in $H$. Write

$$
\begin{gathered}
\xi=\langle T x, x\rangle, \\
h(t)=t x+(1-t) y \quad(0 \leqslant t \leqslant 1),
\end{gathered}
$$

and

$$
a=\operatorname{Re}\langle x, y\rangle, \quad b=\operatorname{Re}\langle T x, y\rangle .
$$

As before, we may assume that $\langle T y, y\rangle=0$. Routine computation with inner products show that

$$
\left\langle T\left(\|h(t)\|^{-1} h(t)\right),\|h(t)\|^{-1} h(t)\right\rangle=c\langle T x, x\rangle+(1-c)\langle T y, y\rangle
$$

if and only if

$$
p(t)=(2 c(1-a) \xi+2 b-\xi) t^{2}-(2 c(1-a) \xi+2 b) t+c \xi=0 .
$$

Since $p$ is continuous and

$$
p(0) p(1)=c(c-1) \xi^{2} \leqslant 0,
$$

we see from the foregoing remark about the intermediate value theorem that LLPO implies the existence of $t \in[0,1]$ such that equation (4) holds. Hence LLPO implies that we can remove the hypothesis $\langle T x, x\rangle \neq\langle T y, y\rangle$ from Theorem 1 .

The following converse shows that we cannot hope to improve, constructively, on Theorem 1 and the extension discussed in the preceding section.

Proposition 14. The following statement implies LLPO.

If $T$ is a selfadjoint operator on a 2-dimensional complex Hilbert space $H$, if $x, y$ are unit vectors in $H$ such that $c\langle T x, x\rangle+(1-c)\langle T y, y\rangle$ belongs to $W(T)$ for each $c \in[0,1]$, and if

$$
h(t)=t x+(1-t) y \quad(0 \leqslant t \leqslant 1),
$$

then for each $c \in[0,1]$ there exists $t \in[0,1]$ such that

$$
\left\langle T\left(\|h(t)\|^{-1} h(t)\right),\|h(t)\|^{-1} h(t)\right\rangle=c\langle T x, x\rangle+(1-c)\langle T y, y\rangle .
$$


Proof. Let $H$ be a 2-dimensional Hilbert space, and $x, y$ orthogonal unit vectors in $H$. Given a binary sequence $\left(a_{n}\right)$ with at most one term equal to 1 , let

$$
\xi=\sum_{n=1}^{\infty} \frac{a_{2 n}+a_{2 n+1}}{n^{2}}
$$

With $K_{n}$ as in Lemma 13, define a selfadjoint operator $T$ on $H$ by

$$
\begin{aligned}
& T y=\left(\sum_{n=1}^{\infty} a_{2 n} \xi^{2}+\sum_{n=1}^{\infty} a_{2 n+1} K_{n} \xi\right) x, \\
& T x=\xi x+\left(\sum_{n=1}^{\infty} a_{2 n} \xi^{2}+\sum_{n=1}^{\infty} a_{2 n+1} K_{n} \xi\right) y .
\end{aligned}
$$

Given $c \in[0,1]$, consider the problem of finding a unit vector $z \in H$ such that

$$
\langle T z, z\rangle=c\langle T x, x\rangle+c\langle T y, y\rangle=c \xi .
$$

Writing $z=\lambda y+\mu x$, with $|\lambda|^{2}+|\mu|^{2}=1$, we reduce equation (5) to

$$
|\mu|^{2} \xi+2 \operatorname{Re}\left(\lambda \mu^{*}\left(\sum_{n=1}^{\infty} a_{2 n} \xi^{2}+\sum_{n=1}^{\infty} a_{2 n+1} K_{n} \xi\right)\right)=c \xi,
$$

which is satisfied by taking $\lambda=\sqrt{1-c}$ and $\mu=\mathrm{i} \sqrt{c}$. Thus $c\langle T x, x\rangle+(1-c)\langle T y, y\rangle$ belongs to $W(T)$.

Next, writing $b=\operatorname{Re}\langle T x, y\rangle$ and taking $c=\frac{1}{2}$, we see that equation (5) becomes

$$
2 b t^{2}-(\xi+2 b) t+\frac{1}{2} \xi=0,
$$

which, if $\xi \neq 0$, can be rewritten

$$
\frac{2 b}{\xi} t^{2}-\left(1+\frac{2 b}{\xi}\right) t+\frac{1}{2}=0 .
$$

If $a_{2 N}=1$, then equation (6) becomes

$$
2 \xi t^{2}-(1+2 \xi) t+\frac{1}{2}=0,
$$

whose only solution in $[0,1]$ is

$$
t_{-}=\frac{1+2 \xi-\sqrt{1+4 \xi^{2}}}{4 \xi} .
$$

On the other hand, if $a_{2 N+1}=0$, then equation (6) becomes

$$
2 K_{n} t^{2}-\left(1+2 K_{n}\right) t+\frac{1}{2}=0,
$$

whose only solution in $[0,1]$ is

$$
t_{-}=\frac{1+2 K_{n}-\sqrt{1+4 K_{n}^{2}}}{4 K_{n}} .
$$

We now proceed as follows. First, noting that

$$
\lim _{\xi \rightarrow 0^{+}} \frac{1+2 \xi-\sqrt{1+4 \xi^{2}}}{4 \xi}=\frac{1}{2},
$$


we find $r>0$ such that if $|\xi|<r$, then

$$
\frac{1+2 \xi-\sqrt{1+4 \xi^{2}}}{4 \xi} \geqslant \frac{3}{8} \text {. }
$$

Secondly, noting that

$$
\lim _{n \rightarrow \infty} \frac{1+2 K_{n}-\sqrt{1+4 K_{n}^{2}}}{4 K_{n}}=0,
$$

we compute a positive integer $N$ such that

$$
\frac{1+2 K_{n}-\sqrt{1+4 K_{n}^{2}}}{4 K_{n}}<\frac{1}{8}
$$

for all $n \geqslant N$. Now suppose that equation (4) has a solution $t=\tau \in[0,1]$, and consider $a_{N}$. If $a_{k}=1$ for some $k \leqslant N$, then either $a_{n}=0$ for all even $n$ or else $a_{n}=0$ for all odd $n$; so we may assume that $a_{k}=0$ for all $k \leqslant N$; we may also assume that $|\xi|<r$. Either $\tau>\frac{1}{8}$ or $\tau<\frac{3}{8}$. Consider the first case, and suppose that $a_{2 n+1}=1$ for some $n$ with $2 n+1>N$. Then $b=K_{n} \xi \neq 0$, so

$$
\tau=\frac{1+2 K_{n}-2 \sqrt{1+4 K_{n}^{2}}}{4 K_{n}}<\frac{1}{8} c,
$$

a contradiction; hence $a_{k}=0$ for all odd $k>N$ and therefore for all odd $k$. Now consider the case $\tau<\frac{3}{8}$, and suppose that $a_{2 n}=1$ for some $n$ with $2 n>N$. Then $b=\xi^{2} \neq 0$, and

$$
t=\frac{1+2 \xi-\sqrt{1+4 \xi^{2}}}{4 \xi} \geqslant \frac{3}{8},
$$

since $|\xi|<r$; this contradiction ensures that $a_{k}=0$ for all even $k>N$ and therefore for all even $k$.

Finally we consider the possibility of improving Theorem 2. On inspection of the proof of that theorem, we see that the convexity of $W(T)$ can be established, provided that, for any complex number $z$, we can find a complex number $\gamma$ such that $|\gamma|=1$ and $\operatorname{Re} \gamma z=0$. We can do this with the aid of LLPO as follows.

Under LLPO, either $\operatorname{Re} z \geqslant 0$ or $\operatorname{Re} z \leqslant 0$. Thus the continuous function $f$ defined on $[0, \pi]$ by $f(t)=\operatorname{Re}\left(\mathrm{e}^{\mathrm{i} t} z\right)$ satisfies $f(0) f(\pi) \leqslant 0$. By the remarks on the intermediate value theorem at the beginning of this section, there exists $\tau \in[0, \pi]$ such that $f(\tau)=0$; so $\operatorname{Re} \gamma z=0$ where $\gamma=\mathrm{e}^{\mathrm{i} \tau}$.

To prove that Theorem 2 cannot be improved constructively, it is enough to show that the proposed improvement would entail $\mathrm{LLPO}_{3}$. For this we need one more lemma.

Lemma 15. If $\lambda, \mu$ are complex numbers satisfying the equations

$$
\begin{array}{r}
4 \operatorname{Re}\left(\lambda \mu^{*}\right)+2|\mu|^{2}=1, \\
|\lambda|^{2}+|\mu|^{2}=1,
\end{array}
$$

then $|\lambda| \geqslant \frac{1}{5}$ and $|\mu| \geqslant \frac{1}{5}$.

Proof. If $|\mu|<\frac{1}{5}$, then $\left|4 \operatorname{Re}\left(\lambda \mu^{*}\right)\right|<\frac{4}{5}$ and so

$$
4 \operatorname{Re}\left(\lambda \mu^{*}\right)+2|\mu|^{2}<\frac{4}{5}+\frac{2}{25}<1,
$$


a contradiction; whence $|\mu| \geqslant \frac{1}{5}$. On the other hand, if $|\lambda|<\frac{1}{5}$, then $\left|4 \operatorname{Re}\left(\lambda \mu^{*}\right)\right|<\frac{4}{5}$ and so

$$
\begin{aligned}
4 \operatorname{Re}\left(\lambda \mu^{*}\right)+2|\mu|^{2} & >2\left(1-|\lambda|^{2}\right)-\frac{4}{5} \\
& >2\left(\frac{24}{25}\right)-\frac{4}{5} \\
& >1,
\end{aligned}
$$

a contradiction. Hence $|\lambda| \geqslant \frac{1}{5}$.

Proposition 16. The following statement implies $\mathrm{LLPO}_{3}$.

The numerical range of a selfadjoint operator on a 2-dimensional complex Hilbert space is convex.

Proof. Let $H, x, y,\left(a_{n}\right)$, be as in the proof of Proposition 14. Define

$$
\xi=\sum_{n=1}^{\infty} \frac{a_{3 n}+a_{3 n+1}+a_{3 n+2}}{n^{2}},
$$

and define a selfadjoint operator $T$ on $H$ by

$$
\begin{aligned}
& T y=\left(\sum_{n=1}^{\infty} a_{3 n} \mathrm{i} \xi+\sum_{n=1}^{\infty} a_{3 n+1} \mathrm{i} n \xi+\sum_{n=1}^{\infty} a_{3 n+2} n \xi\right) x, \\
& T x=\xi x+\left(-\sum_{n=1}^{\infty} a_{3 n} \mathrm{i} \xi-\sum_{n=1}^{\infty} a_{3 n+1} \mathrm{i} n \xi+\sum_{n=1}^{\infty} a_{3 n+2} n \xi\right) y .
\end{aligned}
$$

Consider the problem of finding a unit vector $z=\lambda y+\mu x$ satisfying

$$
\langle T z, z\rangle=\frac{1}{2}\langle T x, x\rangle+\frac{1}{2}\langle T y, y\rangle=\frac{1}{2} \xi
$$

which reduces to

$$
|\mu|^{2} \xi+2 \operatorname{Re} \lambda \mu^{*}\left(\sum_{n=1}^{\infty} a_{3 n} \mathrm{i} \xi+\sum_{n=1}^{\infty} a_{3 n+1} \mathrm{i} n \xi+\sum_{n=1}^{\infty} a_{3 n+2} n \xi\right)=\frac{\xi}{2} .
$$

If $a_{3 N+1}=1$, we must solve the equations

$$
\begin{aligned}
2|\mu|^{2}-4 N \operatorname{Im}\left(\lambda \mu^{*}\right) & =1, \\
|\lambda|^{2}+|\mu|^{2} & =1 .
\end{aligned}
$$

We then have

$$
\left|\operatorname{Im}\left(\lambda \mu^{*}\right)\right|=\left.\frac{1}{4 N}|1-2| \mu\right|^{2} \mid \leqslant \frac{3}{4 N} .
$$

If $a_{3 N+2}=1$, we must solve the equations

$$
\begin{aligned}
2|\mu|^{2}+4 N \operatorname{Re}\left(\lambda \mu^{*}\right) & =1, \\
|\lambda|^{2}+|\mu|^{2} & =1 .
\end{aligned}
$$

In this case, $\left|\operatorname{Re}\left(\lambda \mu^{*}\right)\right| \leqslant \frac{3}{4 N}$. If $a_{3 N}=1$, we must solve the equations

$$
\begin{aligned}
2|\mu|^{2}-4 \operatorname{Im}\left(\lambda \mu^{*}\right) & =1, \\
|\lambda|^{2}+|\mu|^{2} & =1 .
\end{aligned}
$$


In this case we must have $|\lambda| \geqslant \frac{1}{5}$ and $|\mu| \geqslant \frac{1}{5}$, by Lemma 15 (applied with $\lambda$ replaced by $-\mathrm{i} \lambda)$.

Now suppose that we have found a unit vector $z=\lambda y+\mu x$ satisfying equation (5) with $c=\frac{1}{2}$. Either $\left|\lambda \mu^{*}\right|<\frac{1}{25}$ or $\lambda \mu^{*} \neq 0$. In the first case we see from the foregoing arguments that $a_{n}=0$ for each $n$. In the second case either $\operatorname{Re}\left(\lambda \mu^{*}\right) \neq 0$ or $\operatorname{Im}\left(\lambda \mu^{*}\right) \neq 0$. To handle the first of these alternatives, we choose $v$ such that $\frac{3}{4 v}<\left|\operatorname{Re}\left(\lambda \mu^{*}\right)\right|$. If $a_{3 n+1}=1$ for some $n>v$, then $\left|\operatorname{Re}\left(\lambda \mu^{*}\right)\right| \leqslant \frac{3}{4 n}<\frac{3}{4 v}$, a contradiction. It follows that if $a_{3 n+1}=0$ for all $n \leqslant v$, then $a_{3 n+1}=0$ for all $n$. Hence

$$
\forall n\left(a_{3 n}=0\right) \vee \forall n\left(a_{3 n+1}=0\right) \vee \forall n\left(a_{3 n+2}=0\right) .
$$

Finally, assuming that $\operatorname{Im}\left(\lambda \mu^{*}\right)=0$, and choosing a positive integer $v$ such that $\left|\operatorname{Im}\left(\lambda \mu^{*}\right)\right|>$ $\frac{3}{4 v}$, we see that if $a_{3 n+2}=0$ for all $n \leqslant v$, then $a_{3 n+2}=0$ for all $n$, whence equation (7) holds in this case also.

Acknowledgement. The authors wish to thank the Arthington-Davy Fund for providing the scholarship that supported Robin Havea during the writing of this paper.

\section{References}

1. ERrett Bishop and Douglas Bridges, Constructive analysis, Grundlehren Math. Wiss. 279 (Springer-Verlag, Heidelberg, 1985). 192, 193

2. Douglas Bridges, 'Constructive mathematics: a foundation for computable analysis', Theoretical Comput. Sci. 219 (1999) 95-109. 192

3. Douglas Bridges and Hajime Ishihara, 'Locating the range of an operator on a Hilbert space', Bull. London Math. Soc. 24 (1992) 599-605. 198

4. Douglas Bridges and Fred Richman, Varieties of constructive mathematics, London Math. Soc. Lecture Notes 97 (Cambridge University Press, 1987). 192, 192, 193, 200

5. Douglas Bridges, Fred Richman and Peter Schuster, 'Adjoints, absolute values, and polar decompositions', J. Operator Theory, to appear. 191

6. Paul R. Halmos, A Hilbert space problem book, Grad. Texts in Math. 19 (SpringerVerlag, Heidelberg, 1974). 191, 198

7. Hajime Ishihara, 'Continuity and nondiscontinuity in constructive mathematics', $J$. Symbolic Logic 56 (1991) 1349-1354. 197

8. B. A. Kushner, Lectures on constructive mathematical analysis (Amer. Math. Soc., Providence, RI, 1985). 192

9. Fred Richman, 'Intuitionism as generalization', Philos. Math. 5 (1990) 124-128. 192

10. Fred Richman, 'Polynomials and linear transformations', Linear Algebra Appl. 131 (1990) 131-137. 201

11. A. S. Troelstra and D. van Dalen, Constructivism in mathematics: an introduction, two volumes (North Holland, Amsterdam, 1988). 192, 192

12. Klaus Weinrauch, 'A foundation for computable analysis', Combinatorics, complexity, \& logic, (ed. D. S. Bridges, C. S. Calude, J. Gibbons, S. Reeves and I.H. Witten) Conference Proc., Auckland, 9-13 December 1996 (Springer-Verlag, Singapore, 1996). 192 
A constructive analysis of a proof that the numerical range is convex

Douglas Bridges d.bridges@math.canterbury.ac.nz

Robin Havea rha40estudent. canterbury.ac.nz

Department of Mathematics and Statistics

University of Canterbury

Private Bag 4800

Christchurch 1

New Zealand 